\title{
LA STRUTTURA DELL'ATLANTICO (*)
}

\author{
J. P. Rothé
}

Lo studio della struttura dell'Atlantico solleva un problema, che ha gia att:rato l'atenzione di numerosi ricercatori. I lettori di "Annali di Geofisica " hamuo di recente potuto rendersi conto in due riprese dei lavori dei nostri colleghi italiani P. Caloi, L. Marcelli e G. Pannocchia (1)-(2): le ricerche di questi autori si propongono di cimentare la "ipotesi di lavoro" da me sviluppata dinanzi al congresso delle Societés Savantes francesi, riunite a Strasburgo nell'aprile 1947 e pubblicata successivamente in una nota presentata il 2 aprile 1947 alla Académie de Sciences di Parigi (3).(4). Ritengo opportuno ricordare questa ipotesi nel presente lavoro ed apportare, a mia volta, alcuni rilievi sui risultati, ottenuti dai nostri colleghi italiani, insieme con nuovi argomenti ricavati dai lavori recenti.

1. - ì noto che la osservazione della analogia fra i contorni delle coste americana e africana delloceano Atlantico spinse Wegener alla formulazione della sua teoria sulla deriva dei continenti. Orat, dopo avere esaminato numerosi fatti d'ordine sismologico o morfologico, sono stato indotto a proporre la seguente ipotesi.

L'Oceano Atlantico si divide in due domini: uno dei quali, a est della crestn mediana, e un dominio a strultura costiNextal.e. collegantesi col blocco africano; invece la parte occidentale del dominio atlantico è, con tutta probabilitì, a struthura pacirica, il che significa che esso è costituito esclusiramente di sima.

In queste condizioni, il parallelismo fra le coste africana e americana, invocato da Wegener, du Toit, ecc. deve, in realti, essere ricercato fra la costa americana e la cresta mediana dell'Atlantico, le cui forme generali sono le stesse (fig. 1). Se deriva e'è stata, essa si è verificata unicamente sulla distanza cresta mediana-costa americana. La cresta mediana atlantica e la sua zona sismica corrispondono al rilievo limite del blocco africano, che viene a urtare lo zoccolo a struttura simatico, costituente la parte occidentale dell Atlantico.

Il vero Oceano Atlantico è la ampia fessura aperta a ovest della cresta mediana fra i blocchi continentali eurafricano e americano.

Lipotesi proposta toglie lobbiezione alla deriva dei continenti, riguardante l'esistenza sempre più probabile di un continente Atlantide, affondato tra le Azzorre e il distretto di Gibilterra. Essa spiega inoltre come, quando si misura la velociti media delle onde sismiche attraverso R.talatico, considerato come un tutto. si trova un valore intermedio fra il valore della velociti al di sotto del Parifico e quello al di sotto dei continenti.

(*) Traduzione in lingua italiana della Nota di pag. 27. 
2. - I fatti sismologici e morfologici, che nel 1917 avevano attirato la mia attenzione, possono riassumersi nel modo seguente:

I. - Particolarmente istruttivo $\grave{e}$, anzitutto, lo studio dellat distribuzione degli epicentri sullintero globo, quale può stabilirsi in base alle determinazioni eseguite, gia da molti anni, da parte del Bureau International de Séismologie di Strasburgo e ai lavori, tra i molti su questo argomento, di Gutenberg e Richter (i)) da un tale esame si desume lesistenza di grandi blocchi stabili, limitati da zone sismiche attive; fra questi grandi rilievi si stacca un blocco assai più esteso di quello attualmente definito dalle carte geografiche (fig. 2). Questo blocco africano è limitato a ovest dalla zona sismica, che riguarda la cresta mediana sottomarina dell'Atlantico, mentre dal blocco Europa-Asia lo separa la zona sismica alpina: quest'ultima zona, che altraversa il dominio della Mesogea, segue, toccando il Nord Africa, lWuropa e l'Asia, le linee dei corrugamenti alpini (Himalaya, Caucaso, Carpazi, Alpi. Ap)pennini, Atlante, Cordigliera Betica) e raggiunge la zona sismica mediana dell Atlantico nei dintorni delle Azzorre (†). La raggiunge ma non la attraversa. Contrariamente a quanto si riteneva non esiste alcun indizio di sismiciti fra la cresta alantica e l'arco delle Antille, il quale, a sua volta, fa parte del cerchio sismico circumpacifico. Cosi pure la carta degli epicentri mostra che la geosinclinale della Mesogea non attraversa l'Atlantico da parte a parte, né raggiunge la geosinclinale parifica, come appare ancora su certe carte classicle della struttura complessiva del Globo.

II. - Lynch (6) hat segnaliato che nel terremoto del 11 settembre 19.15, prodoltosi sulla cresta mediana atlantica (epicentro $7^{\circ}, 0 \mathrm{~N}: 38^{\circ} .8 \mathrm{~W}$, la velocita delle onde superficiali di Love, misuratit all'osservatorio di Fordlam (New-York), distante $5100 \mathrm{~km}$ dall'epicentro, era di $4,4 \mathrm{~km}$ al secondo per un periodo di 23 secondi. Si tratta di una velociti indubbiamente confrontabile e anche un po' superiore al quella dell'onda di Love dello stesso periodo sotto il Pacifico.

III. - Il mio collega G. Dubois, professore di Geologia all'Lniversita di Strasburgo, con il quale mi sono frequentemente intrattenuto riguardo al problema così attraente della struttura terrestre, mi ha segnalato linteressinte risultato, ottenuto con i rilievi batimetrici, recentemente eseguiti nell Atlantico. Essi dimostrano cle l'Atlantico Sud presenta, ad est della cresta mediana, una successione di bacini e di creste orientate SW-NE, la quale prolunga le unita morfologicle del continente africano. Queste uniti sono riportate in fig. 1 in base alla carta pubblicata da J. H. F. Umbgrove (T).

IV. - E noto che la costa orientale degli Stati Uniti e delle provincie marittime del Canada presenta un carattere moriologico del tipo pacifico li rilievi sono allineati parallelamente alla costa); invece le coste spagnole e bretoni sono coste a rias di tipo atlantico.

V. - Gli archi delle Antille del Nord (Portorico, Martinica, Guadalupai, ece) e delle Antille del Sud (Georgia del Sud, Sandwich, Oreadi del Sud, ecc.) oceupano in rapporto al bordo occidentale dell'Atlantico, la stessa posizione (fig. 1) delle

(-) V. p. es. la carta al p. 22.73 in: J. P. Rotué, Séismes el Volcans iCollezione "Que sais.je?"). Parigi, 1946. 
Hhirlande insulari (Curili, Marianne, ecc.) in rapporto al bordo occidentale del Pacilico. L'analogia è confermata dalla scopertil, dovutit a $F$. Vening-Meinesz, dell'asse delle anomalie negative della gravità, che prolunga dal lato dell'Atlantico l'arco delle Antille.

3. - Questa ipotesi è statia da me avanzata con la speranza di stimolare le ricerche, cosicché io ho letto col più vivo interesse i lavori dei nostri colleghi italiani sopra menzionati $(1,2)$ : d'altro canto il secondo di questi due articoli risponde già parzialmente a una nota personale da me comunicata al prof. Caloi nell'aprile 1950, lo sviluppo della quale costituisce la presente memorial.

Purtutavia, dinanzi ai fatti apportati, sono un po' meravigliato delle conclusioni formali alle quali arrivano questi autori. Essi mi permetteranno di presentare qui alcune osservazioni. La loro riprova della mia ipotesi si basa sul calcolo della velocita di propagazione delle onde superficiali e in particolare delle onde di Love attraverso l'Atlantico a partire da un epicentro situato solto la cresta mediana a $8^{\prime \prime}\left(2^{\prime}\right.$ di latitudine nord e $38^{\prime \prime} 4^{\prime}$ di longitudine ovest. Rivolgerò l'attenzione sui punti seguenti:

I. - La fig. 8, pubblicata dal prof. Caloi e coll. (1, pag. 358), la quale riassume i risultati delle misure, mostra con chiarezza - in una maniera quasi insperata! - la separazione delle stazioni in due gruppi (fig. 3 ).

a) il gruppo delle stazioni americane, situate in prossimita dell'Alantico (San Juan, Bermuda, Halifax, Harvard, Ottawa): le velocita misurate sono assai forti;

b) Il gruppo delle stazioni europee, al quale si aggiunge in maniera assai caratteristica la stazione di Tananarive: le velocita sono molto più deboli con periodi uguali.

In questa classificazione rigorosa compare un'unica anomalia: la grande velocità riscontrata alla stazione di Lisbona (distanza $H+80 \mathrm{~km}$ ). Si osserverà che per questa stazione i treni d'onda SS e SSS devono emergere rispettivamente a $19^{\mathrm{li}} 51^{\mathrm{m}} 34^{\mathrm{s}}$ . 19h $52^{\prime \prime \prime} 18^{*}$; a questa distanza essi posseggono gia un periodo relativamente grande ed hanno potuto mascherare l'arrivo delle onde di Love. Se, in conseguenza di tale interferenzal, questo arrivo è stato letto troppo presto si spiegherebbe lanomalia positiva della velocità misuratia a Lisbona.

II. - Se, dunque, eliminiamo questa unica anomalia, la differenza di velocita, a seconda che le onde superficiali si sono propatgate attraverso la metà occidentale o attraverso la metà orientale dell Atlantico, è notevole. Ed è tanto più notevole in quanto per raggiungere le stazioni americane le onde devono assolutamente attraversare una pialtaforma epicontinentale Il Appalachi sommerso e la sua copertural grande da 200 a $300 \mathrm{~km}(\mathrm{~s}$.

Si puó confrontare, p. es., la lunghezzal dei percorsi continentali e oceanici seguiti dalle onde per raggiungere Ottawa a ovest e Jersey, Tortosia, Barcellona a est. Si trova (*):

(*) Distanze misurate sul mippamondo. 
percorso oceanico

$\begin{array}{ll}\text { Ottawal } & 4.600 \mathrm{~km} \\ \text { Jersey } & 5.300 \\ \text { Tortosa } & 4.300 \\ \text { Barcellona } & 4.500\end{array}$

percorso continentale

$800 \mathrm{~km}$

400

1.000

1.000

Ora la velocità media delle onde di Love è maggiore di $0, \mathrm{k} \mathrm{km} / \mathrm{sec}$ a Ottawa che nelle stazioni europee, quantunque $i$ percorsi oceanico e continentale siano assolutamente dello stesso ordine di grandezza: il percorso continentale, seguito dall'onda, che raggiunge Jersey, è sempre il più corto.

S̋intende che, come è gì stato rilevato dagli autori italiani, bisogna eliminare dal confronto le stazioni americane di Sitkil, Victoria, Pasadena ecc. alle quali le onde sismiche arrivano dopo avere attraversato tutto il continente americamo.

III. - Per quel che riguarda la stazione di Tananarive gli autori italiani scrivono: "Nel quadrante sud orientale le osservazioni sismiche sono limitate a quelle della stazione di Tamanarive. La relativamente elevata velocità di propagazione osservata per le onde superliciali registrate in della stazione, lascia peró ritenere che il tragitto atlantico di dette onde ha interessato zone ad elevatissima velocita per le onde superficiali ".

Purtuttavia la figura 3 mostra chiaramente che il valore di $4,0 \mathrm{~km} / \mathrm{sec}$ registrato a Tananarive si allinea perfettamente sulla retti, che si può tirare per $i$ punti rappresentativi dei valori misurati nelle stazioni situate ad est della cresta mediana (cerchietti vuoti). Nonostante le onde seguano un percorso oceanico di $6000 \mathrm{~km}$ e un percorso continentale di $4000 \mathrm{~km}$, la loro velocità è completamente analoga a quelle misurate a Barcellona, Tortosa e Copenhagen. Riteniamo quindi che tale concordanza confermi l'omogeneita della costituzione, esistente ad est della cresta mediana atlantica, qualunque sìa la natura del percorso visibile, oceanico o continentale.

IV. - Ci permetteremo di confutare ladozione da parte degli autori italiani della carta, che essi presentano in fig. 11, a sostegno dei loro argomenti.

Sta di fatto che B. Gutenberg e Richter (") hanno segnalato in numerosi articoli, che una carta come quella riprodotta in figura 11 , lat quale $\grave{e}$ batsata su tutte le determinazioni epicentrali calcolate nell'International Scismological Summary, fornisce unidea necessariamente falsafa per quel che riguarda la densith degli ossercatori. La concentrazione degli epicentri siomici sulle Alpi, in Italia e nei Ballani è erronea: essil è dovuta allia rilevante densita degli osservatori sismici nell'Europa occidentale. Proprio allo scopo di ottenere una immagine più veridica della sismiciti nelle differenti parti della terra, C. Richter ha introdotto la feconda nozione di magnitudo.

Daltro canto gli autori americani nella loro opera recente confermano lopinione gia in precedenzal enunciata: "Seismic data support a continuation of the active beh into the Atlantic as far as the Agores but not farther. There is no justification for extending it to the West Indies, as was done on some early seimmic milps of the world (5, p. 71$)$.

Questa zona sismica è interessata in particolare dall'epicentro del grande terremoto (probabilmente di magnitudo 9), detto “di Lisbonal (1755) e di quello 
della scossa (di magnitudo 8,3 ) del 25 novembre 19.4l, clie provocó la rottura dei cavi sottomarini Brest-Casablanca e Brest-I)akar.

In base allopera degli autori americani la lista degli epicentri marini nella zona compresal fra il $10^{\prime \prime}$ e il $32^{\prime \prime}$ grado di longitudine ovest può staluilirsi come scgue :

\begin{tabular}{|c|c|c|c|}
\hline \multicolumn{4}{|c|}{ Coordinate } \\
\hline Data & Latt. & Long. & Magnitudo o classe \\
\hline $27-12-19+1$ & $36^{\prime \prime} \mathrm{N}$ & $105 \mathrm{~W}$ & $63 / 1$ \\
\hline $11 \cdot 7-1915$ & $37^{\prime \prime}$ & 1045 & $61 / 4$ \\
\hline 7. $3-1930$ & $32^{\prime \prime}$ & $11^{15} 5$ & $d$ \\
\hline $20 \cdot 5-1931$ & $37 \times 5$ & $16^{\prime \prime}$ & 7,1 \\
\hline $25-11-19+1$ & 3705 & $18 " 5$ & 8,3 \\
\hline $29-5 \cdot 19 \cdot 12$ & $38^{\circ}$ & $19^{\circ}$ & $d$ \\
\hline $21-10-1930$ & $36^{4 \prime 5}$ & $23^{\prime \prime}$ & $d$ \\
\hline $8-5-1939$ & $37^{\prime \prime}$ & 2.45 & 7,1 \\
\hline $15 \cdot 8 \cdot 1933$ & $38^{\prime \prime}$ & $26 " 5$ & $d$ \\
\hline $31-8-1926$ & 385 & $28^{\prime \prime}$ & $d$ \\
\hline 5. 1.1926 & $39^{\prime \prime}$ & 290 & 6 \\
\hline $9-7.1931$ & $40 \cdots 2$ & 2905 & $d$ \\
\hline 18. $7-1923$ & $42^{\prime \prime}$ & $29 n 5$ & $d$ \\
\hline 9. 7.1926 & $18^{\prime \prime}$ & 30 & $d$ \\
\hline $27-8.192 .1$ & 1145 & $30 \cdots 5$ & $d$ \\
\hline 19. $7-19.11$ & 385 & $32^{\prime \prime}$ & $d$ \\
\hline
\end{tabular}

Del resto in questa lista figura solo un certo numero di scosse (di classe $d$ o delle (lassi superiori) citate "because their epicenters contribute to seismogeographic information $)$.

Mi à apparso sempre degno di nota il fatto che questa zona sismiea non attraversa la parte occidentale dell Atlantico. Si osservi fra laltro che gli epicentri, indicati da Miss E. Bellamy (l, carta di fig. 11) a ovest della cresta mediana Atlantica, sono dubbi e realmente imprecisi: dopo la revisione essi sono stati eliminati da B. Gutenlerg e C. F. Richter.

V. - E accertato che dalle carte batimetriche appare evidente l'esistenza a F. della cresta atlantica di fosse profonde $6000 \mathrm{~m}$ e che sotto questo punto di vista sembra sicura lanalogia nella struttura superficiale tra le due parti dell'Atlantico. Ilo gia rilevato poc'anzi come le misure recenti mostrino, purtuttavia, alcuni corrugamenti nella parte orientale dell’oceno Atlantico, i quali prolungano gli assi strutturali del continente africano (1, p. 220). Qui aggiungeró solamente che non bisogna dimenticare come una catena corrugata, quale quella delle $\mathrm{Al}_{\mathrm{p}} \mathrm{i}$ (per es.), si immerge fino a 50 o $60 \mathrm{~km}$. di profondita, a una profondità, cioè, 10 volte più grande di quella delle fosse segnalate poco fa. Certamente i fenomeni geologici, che dobbiamo considerare nella separazione del dominio atlantico in due parti, interessano tutto lo spessore della crosta terrestre. Ad ogni modo il lavoro dettagliato dei nostri colleghi italiani conferma quello che si poteva prevedere in base alle misure isolate e cioè il valore elevato della velocità delle onde superficiali propagantesi sotto l'Atlantico occidentale, il cui sottosuolo è dunque con tuttal pro. babilità a composizione simatica. 
4. -- Riassumerò qui alcuni lavori recenti dei quali ho avuto notizia dopo lesposizione preliminare della mia ipotesi.

I. - È nota la difficoltà a cui si va incontro, soprattutto se si tiene conto delle ipotesi di Wegener, nella interpretazione della esistenza della cresta sottomarina sotto MAtlantico, cresti, che le nuove misure batimetriche hamno dimostrato essere composta di catene montane parallele $(10)$.

Ora, dopo la comparsa della mia prima not:i, l'eminente geologo francese L. Glangeatud (11), ha pubblicato una interessante memoria nella quale egli spiegal la formazione delle catene montine attraverso cicli termodinamici sialo-simici. In particolare egli scrive: "Dans toutes les chaines qui se forment ainsi au contact du bloc sialique continental et du sima sous-océanique on peut admettre un rôle plus ou moins accentue de la palingénèse crustale. Notre hypothèse explique pourquoi de telles chaines liminaires, de direction méridienne, jalonnent la bordure du Pacifique, en Amérique comme en Asie. Elles appiraissent partout où les malsses sialiques continentales siffrontent avec le sima, qui occupe une partie du fond du Pacifique. Les phénomènes des chaines liminaires deivent ainsi continuer de nos jours, à la limite du sial et du simal, au Japon, en Californie, sur la bordure orientale des Antilles, et le long de la crète atlintique.

Or J. P. Rothé a émis l'hypothèse que la crète atlantique est située à la limite du bloc sialique africin et du fond simique de l'ouest-Allantique. Elle serait ainsi placée dans les conditions de formation des chaines liminaires sialo-simiques ".

$\dot{E}$ evidente come la mia ipotesi elimina una grossa difficolti, riconducendo al caso generale l'interpretazione di un accidente strutturale tanto importante, la cui posizione era apparsa sempre cosi sorprendente.

Molto probabilmente lungo la cresta mediana atantica si devono verificare alcuni fenomeni di corrugamento e di salita di magni basaltici viscosi, iniettantisi attraverso la zona granitica e gli strati sedimentari; tali fenomeni sarebbero confrontabili con quelli, descritti e spiegati da L. Glangeaud, i quali compaiono nell'Atlinte telliano (Africa del Nord), rilievo che costituisce il confine del blocco africano a contatto con la Mesogea ( 11 bis). Il bacino centrale del Mediterraneo, sprovvisto di attiviti sismical, ci appare come un lago, o per meglio dire un affioramento di sima in mezzo alle masse sialiche eurasiatica e africana.

II. - Le informazioni sulla natura geologica dei fondi dell'Atlantico mancino quasi completamente. Si può pertanto menzionare già qualche lavoro.

In una poetica conferenza P. Termier (12) segnalava la scoperta fatta nel 1898 da parte di un naviglio, impiegato per la posa di un cavo sottomarino: a $4700^{\prime} \mathbf{~}$, $27^{\circ} 20^{\prime} \mathrm{W}$ e ad una profonditi di $3000 \mathrm{~m}$ venne prelevato un frammento di roccia: cra una lava vetrosa a composizione basaltica, la tachilite. Termier pensa che la formazione di questia lava ha dovuto arvenire a pressione atmosferica e, conseguentemente: "la terre qui costitue amjourdhui le fond de l'Atlantique à 900 kilomètres atu nord des $\Lambda$ çores a été recouverte de laves quand elle etait encore emergéen. Le coordinate indicate corrispondono ad un punto della zona sismica della cresta mediana atlantica, al limite fra la zona simatica ed il blocco sialico 'punto l sullia cartal di fig. I). 
Pochi geologi, scriveva Termier, hanno compreso l'importanza di quel campione di tachilite conservato nella École des Mines di Parig̣i.

Numerose pubblicazioni sono state consacrate a giustificare il racconto di Platone riguardinte l'Atlantide, vasto continente (?), isola (?) o penisola (?), popolata da una raza preistorica, che avrebbe invaso l'ovest dell'Europa, dopo che it loro paese era stato sommerso. Gli etnologi collocano questo paese scomparso vicino alle Azzorre o Canarie, considerate da alcuni geologi come il prolungamento del grande Atlante marocchino, oppure vicino alle isole del Capo Verde (13).

Terminando la sua conferenza P. Termier scriveva: "Libre à tous les amoureux des belles légendes de croire à l'histoire platonicienne de l'Atlantide! Non seulement la science, la plus moderne science ne leur en fera pas un crime; mais rest elle-même, qui, par ma voix, les y invite. C'est elle-même qui, les prenant par la main, et les conduisant sur la rive de l'océan fertile en naufrages, évorue à leurs yeux, avec les milliers de nivires désemparés, les continents et les iles sans nombre ensevelis at fond des abimes".

III. -. I nuovi metodi di carotaggio adottati dalle spedizioni oceanografiche svedesi ed americane lasciano sperare che ben presto si entreri in possesso di un numero sempre crescente di campioni di rocce, prelevati nel fondo del mare, sui quali si possino proseguire le ricerche.

Gia ì stato pubblicato un primo risultato dei sondaggi (10). Sono stati prelevati alcuni campioni di calcare dellera cenozoica in due punti della cresta mediana atlanticia. I. Tolstoy ed M. Ewing riferiscono queste scoperte con i seguent? termini: "Dredging the eastern flank of the steep ridge running along the nortlswest edge of the 2100 -- fathom terrace at point $G$ of Plate $?\left(32^{\circ} 30^{\prime} \mathrm{N}, 42^{\circ} 16^{\prime} \mathrm{W}\right)$ brought up a large block of manganese - encrusted limestone of probably Ceno. zoic age ) $(10$, p. 1537$)$.

"At point $\mathrm{E}$ in Plate $2\left(3.4 " 0^{\prime} \mathrm{N}, 30^{\prime \prime} 3^{\prime} \mathrm{W}\right)$ dredging the top yelded a large number of calcareous dises carachterized by peculiar cavities in their center... Thin section of dises reveal the presence of numerous pteropods. According to D. Ericson, all are species that could have inhabited the ocean from the early tertiary to the present. None are sufficiently characteristic of a specific period to date this limestone $)(10$, p. 1537$)$.

E gli autori di queste scoperte concludono:

"One of the big surprises of the 19.17 Atlantis expedition (cruise 150) was the proof of the existence of consolidated Cenozoic limestone on the Mid-Atlantic Ridge. Possibly considerable areas of the Ridge consist of sedimentary rocks. If so, the hypothesis of folded ranges in certain parts of the Ridge should not be rejected arbitrarily, on the sole basis that it is difficult in the light of our scanty knowledge of the history of the earth's crust to explain the why and how of such a process. It must he disproved by experimental and observational data; until then it remains a possibility " $(10$, p. 1339$)$.

Nella nostra carta di fig. 1 i punti $G$ ed $E$ di cui si parla sopra sono designati con le cifre 2 e 3.

IV. - L'estensione alle grandi profondita marine del metodo della prospezione sismica per rifrazione fornisce del pari la possibilita di determinare approssimativamente la struttura degli strati suboceanici.

Nellagosto del 19.19 il Department of Geodesy and Geophrsies della Lniver- 
sita di Cambridge (Inghilterra) ha provato ad applicare il metodo della rifrazione sismica sotto 1300 braccia dacqua nel Nord Atlantico in prossimita del punto di coordinate $53^{\prime \prime} 50^{\prime} \mathrm{N}, 18^{\prime} 40^{\prime} \mathrm{W}$ (punto 4 della nostra carta di fig. 1). I risultati di queste misure sono stati riassunti da M. N. Hill e J. C. Shallow (14):

"The low values for the velocity in the surface-layer (from about $5000 \mathrm{ft} / \mathrm{sec}$ at the surface of the sea bed to a maximum value of approximately $8000 \mathrm{ft} / \mathrm{sec})$ indicate not hightly consolidated marine sedimente... The velocity in the layer below the sediments is similar to those of early Palaeozoic sedimentary rocks or various igneous rocks such as granite $(16.000$ and $17.300 \mathrm{ft}$ /sec). The depht below sealevel of this layer is approximately the same as that of the continental granitic layer, but its thickness, $8.800 \mathrm{ft}$, is considerably less.

The two observations $(21.700 \mathrm{ft} / \mathrm{sec}$ and $20.600 \mathrm{ft} / \mathrm{sec})$ of the velocity in the third type of rock are close to the value assigned to the intermediate layer lying between granitic and ultrabasic layers as determined from near earthquakes ».

Inoltre M. Ewing e i suoi collaboratori (1.), ottengono risultati molto interessanti, ma completamente differenti dai precedenti, pure con il metodo della rifrazione sismica, operando, però, stavolta a 120 miglia a $\mathrm{N} \cdot \mathrm{W}$ di Bermuda in un punto di coordinate $34^{\circ} \mathrm{N}, 66^{\prime \prime} 30^{\prime} \mathrm{W}$ (punto 5 sulla nostra carta di fig. 1 ).

"The ocean floor was found to be composed of two layers. A velocity of $2 . .000$ $\mathrm{ft} / \mathrm{sec}(7,58 \mathrm{hm} / \mathrm{sec})$ was found for the second layer. This was identified with the Pn waves of earthquake seismology attributed to the basaltic layer found below the Mohorovicic discontinuity. A first layer was clearly present, but the velocity was not determined. A velocity of $5.600 \mathrm{ft}$ per sec $(1,70 \mathrm{~km}$ per sec) was assumed, giving a thickness of 4.500 feet for this sedimentary layer. This velocity was chosen from some of our earlier measurements, as yet mostly unpublished. The granitic and intermediate layers are thus absent beneath the ocean floor at this point. The velocity of the basaltic layer compares favorably with those of Slichter in New England and with Jeffreys'world average ".

Citeremo infine le parole di Bucher, che in un meeting del novembre 1949 a El Paso, dichiarava: "that Ewing's conclusions cast doubt on the belief that the floor of the Atlantic Ocean in underlain by a thin "granitic shell". Granitic material may he absent over large part oceans ).

5. - In conclusione basterà riportare sulla carta di fig. 1 la posizione rispettiva dei punti da 1 a 5 : la natura dei terreni che sono stati riconosciuti colà è proprio quella prevedibile in base all"ipotesi da noi formulata nel 1917 e che è riassunto nella seguente tabella:

Punto 1 (Atlantico sialico): roccia vulcanica affondata.

Punto 2 (Atlantico sialico): calcare dellera terziaria.

Punto 3 (Atlantico sialico): calcare dellera terziaria.

Punto 4 (Atlantico sialico): sedimenti e graniti del paleozoiro.

Punto 5 (Atlantico simatico): assenza di granito.

L. Don I.eet (16), nell'intento di confutare le osservazioni di Ewing, gli oppone "in contrast" le misure geofisiche inglesi. Le nostra interpretaione della struttura dell Atlantico spiega immediutamente questo contrusto: la cresta mediunt Atlantica, la cui sismicitì prova che essa è tuttora in evoluzione e che costituisce 
la catena limite del blocco sialico africano, separa le due parti dell'Allantico in due domini a struttura differente.

$$
* *
$$

R. Furon (1i) ha ricordato di recente la scoperta di piastrine di scisti primari a Trilobiti, estratte in un sondaggio effettuato nel 1883 dal "Talisman ) a mezza

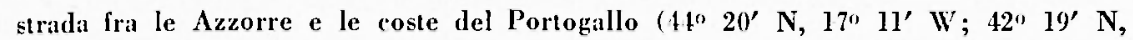
$21^{\circ} 16^{\prime} \mathrm{W}$ ) da una profondita superiore ai $4000 \mathrm{~m}$. "Ce sont, scrive R. Furon, les premier fossiles ramenés des grandes profondeurs. Ils apportent la preuve d'un relief sous-marin partiellement constitué par des roches sédimentaires d’âge paléozoique ".

Per quel che riguarda la parte simatica dellOceano Atlantico la costituzione geologica delle isole Bermude fornisce indicazioni preziose. Esse sono formate da scogli organici costruiti su una catena di picchi vulcanici di direzione NE.SW. Non vi è stata riconosciuta la presenza di terreni sedimentari (1s, 1:1, 20).

\section{RIASSUNTO}

Lautore rammenta la ipotesi di lavoro da lui formulata nel 1947: l'Oceano Allantico e diviso in due domini, una zona occidentale a carattere simatico e unte parte orientale a strutura continentale a est della cresta mediana.

Liautore considera la distribuzione degli ipocentri sismici, la forma delle coste, il prolungamento delle uniti morfologiche africane nel mare e il valore assai eletato della velocitì delle onde sismiche superficinli, propagantesi sotto la parte occidentale dell Atlantico. Discute lo studio di P. Caloi e collaboratori e porta nuovi argomenti a favore della sua ipotesi: dragaggio di una tachilite (Termier) di trilobiti (Furon) di calcari dellera terziaria (Eving e Tolstoy) nella parte orien!ale delldthntico; teoria delle catene liminari (Glangeaud); risultati delle prospezioni sismiche per rifrazione ottenuti ad ovest della Scozia (Hill) e nelle vicinanze dclle Bermule (Ewing). 